\title{
Monte Carlo simulation of uncertainty to identify barriers to optimizing blood pressure control
}

Short title: Simulating uncertainty in hypertension

Lorenzo ZANISI ${ }^{a *}$, Christopher N FLOYD ${ }^{\text {b* }}$, James E BARRETT', Catey BUNCEc ${ }^{c}$, Chris FROHMAIER ${ }^{d}$, Francesco SHANKAR ${ }^{a}$, Phil J CHOWIENCZYK ${ }^{b}$

* These two authors contributed equally to this article and are joint first authors

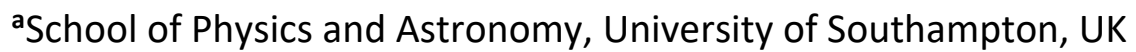

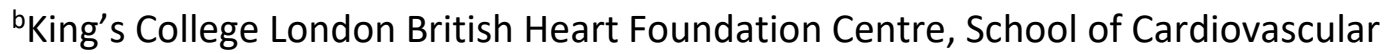
Medicine and Sciences, King's College London, UK

'Department of Population Health Sciences, King's College London, ${ }^{d}$ Institute of Cosmology and Gravitation, Portsmouth University, Portsmouth UK

\section{Correspondence to:}

Phil J CHOWIENCZYK

Clinical Research Facility,

St Thomas' Hospital,

London, SE1 7EH

phil.chowienczyk@kcl.ac.uk

\section{Conflicts of Interest statement:}

The authors declare no conflicts of interest.

Word count including references: 3,706

Number of tables: 1

Number of figures: 4 
Abstract

Objectives: To assess the impact of variable drug response and measurement error on systolic blood pressure (SBP) control.

Methods: We simulated a treat-to-target strategy for populations with different pretreatment SBP, whereby medications were added sequentially until measured SBP $(\mathrm{mSBP})<140 \mathrm{mmHg}$. Monte Carlo simulations determined variability of both drug response (drug eff $\pm \sigma_{\text {drug }} ; 10 \pm 5 \mathrm{mmHg}$ base case) and measurement error ( $\sigma_{\text {meas; }}$; $10 \mathrm{mmHg}$ base case) of true SBP (tSBP). The primary outcome measure was the proportion of individuals who achieved target $<140 \mathrm{mmHg}$.

Results: Decision-making based on mSBP resulted in $35.0 \%$ of individuals with initial tSBP $150 \mathrm{mmHg}$ being either inappropriately given, or inappropriately denied a second drug. When the simulation was run for multiple drug titrations, measurement error limited tSBP control for all populations tested. A strategy of drug titration based on a second measurement for individuals at risk of incorrect decisions (mSBP $120-150 \mathrm{mmHg} ; \sigma_{\text {meas }} 15 \mathrm{mmHg}$ ) reduced the proportion above target from 40.1 to $30.0 \%$ when initial tSBP $160 \mathrm{mmHg}$. When the measurement variability for the second reading was reduced below that usually seen in clinical practice ( $\sigma_{\text {meas }}$ $5 \mathrm{mmHg}$ ), the proportion above target decreased further to $17.4 \%$.

Conclusions: In this simulation, measurement error had the greatest impact on the proportion of individuals achieving their SBP target. Efforts to reduce this error through repeated-measures, alternative measurement techniques or changing thresholds, are promising strategies to reduce cardiovascular morbidity and mortality and should be investigated in clinical trials. Here we have shown that Monte-Carlo simulations are a useful technique to investigate the influence of uncertainty for different hypertension management strategies.

Keywords: Hypertension, Blood Pressure, Monte Carlo Method, Computer Models, Uncertainty, Antihypertensive Agents 


\section{Introduction}

Current medical management of many common conditions involves initiating or changing treatment based on quantitative, semi-quantitative or qualitative thresholds of some measure of response to treatments. The values that define thresholds commonly represent either a surrogate outcome measure (e.g. blood pressure or spirometry) or elements of a diagnostic or symptomatic metric (e.g. inflammatory markers or a depression score). Typically, the focus is upon the average value of a measurements derived from a clinical encounter without attention on dispersion, yet variation or uncertainty in these values due to measurement error and/or physiological variation has the potential to result in erroneous decisions, with significant implications for efficient and effective healthcare delivery. As the evidence-base for personalised medicine increases, treatments and treatment thresholds are likely to be set according to characteristics of the individual and the impact of measurement error for an individual will increase [1].

Here we present an example of how measurement uncertainty impacts hypertension management, a particularly important example as hypertension is the major cause of death and disability worldwide $[2,3]$. Hypertension is particular amenable to a modeling approach for a number of reasons; (i) there is a strong association between the measured variable (blood pressure; BP) and clinical outcomes [4, 5], (ii) guidelines recommend the initiation of treatment based on a threshold BP and subsequent drug titration to achieve a target BP [6, 7], (iii) both drug response and measurement variability are well-described [8-12], and (iv) most individuals require treatment with two or more drugs, thus providing the potential for measurement error to be compounded [11]. Furthermore, whilst short-, medium- and long-term BP variability has been associated with adverse outcomes, it is unclear to what extent measurement error (and hence over- or undertreatment) may contribute [13].

There is an awareness among clinicians that measurement error can result in incorrect treatment decisions and that approaches to minimize error are important [14-16]. Previous studies have used probabilistic simulations to investigate how 
measurement error can impact on the diagnosis of hypertension, with both inadequate device calibration and normal physiological variation contributing to misdiagnosis [17-19]. This study is the first simulation to investigate how measurement error impacts on achieving a blood pressure target for multiple drug titration steps and considers the impact of this on the proportion of individual achieving BP control.

\section{Methods}

We used a Monte Carlo simulation to investigate the impact of drug response and measurement variability on treatment outcomes for populations of hypertensive individuals. Monte Carlo simulations are powerful techniques based on mathematical models that describe the behavior of a system over time in response to stochastic (or random) variability of input parameters [20, 21]. Monte Carlo simulations are widely used in Astrophysics and other physical sciences where understanding the uncertainty arising from measurement error plays an important part in interpreting data[22, 23]

Key parameters in our model were the true systolic BP (tSBP; a hypothetical value made without measurement error or physiological variability) and measured SBP (mSBP) which is a value obtained by the observer and includes varying degrees of error. Each mSBP value represented the outcome of a clinical encounter, for example, the mean of multiple office $\mathrm{BP}$ readings or ambulatory blood pressure measurement. We focused on SBP due to its predictive validity with outcomes, and recent focus in major treat-to-target studies [3, 24-27].

Observed BP variation arises from the sum of measurement error and physiological variation (characterised by the standard deviation of repeated measurements over time, $\left.\sigma_{\text {meas }}\right)$, and by the variation in response to a drug $\left(\sigma_{\text {drug }}\right)$. Total variance can therefore be described as follows:

$$
\sigma_{\text {tot }}^{2}=\sigma_{\text {meas }}{ }^{2}+\sigma_{\text {drug }}^{2}
$$

Values for these parameters and the mean response to drug treatment (drug eff) were extracted from published literature, with a range input into the simulation to explore 
their effects [8-12]. Base case parameters were as follows: $\sigma_{\text {meas }} 10 \mathrm{mmHg}$, drugeff 10 $\mathrm{mmHg}$ and $\sigma_{\text {drug }} 5 \mathrm{mmHg}$.

Populations entered the simulation with one of three pre-treatment (initial) tSBPs: 150, 160 or $170 \mathrm{mmHg}$. Individuals then all received a single drug with response drug $_{\text {eff }} \pm \sigma_{\text {drug }}$ and underwent BP measurement with variation $\sigma_{\text {meas. }}$. Those with $\mathrm{mSBP}$ $<140 \mathrm{mmHg}$ then exited the simulation and received no further drugs, as to the observer they appeared to have achieved target. For those with $\mathrm{mSBP} \geq 140 \mathrm{mmHg}$ (irrespective of tSBP) they progressed to Step 2 and received another drug. This process of drug titration continued for each individual until their $\mathrm{mSBP}<140 \mathrm{mmHg}$. The number of steps for each individual represents the number of drugs received.

The statistical outputs from this simulation were descriptive and considered the proportion of individuals who achieved SBP target $(<140 \mathrm{mmHg})$ according to UK guidelines [27]. Undertreated was defined as tSBP $\geq 140 \mathrm{mmHg}$, controlled as tSBP 120-139 $\mathrm{mmHg}$, and overtreated as tSBP $<120 \mathrm{mmHg}$.

\section{Results}

The typical expected distribution of tSBP and mSBP for our virtual population in response to a single antihypertensive titration is shown in Figure 1. In this example, for an initial tSBP $150 \mathrm{mmHg}$, the use of a drug with drugeff $10 \mathrm{mmHg}$ and $\sigma_{\text {drug }} 5$ $\mathrm{mmHg}$ results in a mean tSBP $140 \mathrm{mmHg}$. As drug effect is normally distributed in our simulation, the tSBP of the population will be evenly distributed. The mean of mSBP values obtained by an observer will also be $140 \mathrm{mmHg}$ and normally distributed. However, the range of mSBP values is much wider than that for tSBP, with approximately $4 \%$ appearing to be 'super responders' with $\mathrm{mSBP}<120 \mathrm{mmHg}$. In reality, $<0.001 \%$ of the simulated population have tSBP $<120 \mathrm{mmHg}$. The use of a single $\mathrm{mSBP}$ reading to determine response to a single drug titration resulted in $35.0 \%$ of the population either being inappropriately given, or inappropriately denied a second drug. Qualitatively similar results were found for other values of initial tSBP. 
The relative effects of the input parameters on the proportion of individuals who appear to achieve target $(\mathrm{mSBP}<140 \mathrm{mmHg}$ ) are presented in Figure 2. Variation in drug $_{\text {eff }}$ had the largest impact on this simulation with the effect greatest at higher initial tSBP. Where initial tSBP was $170 \mathrm{mmHg}$, mSBP $<140 \mathrm{mmHg}$ was achieved in $<20 \%$ for three drugs when drug response was $5 \pm 5 \mathrm{mmHg}$, compared to $>90 \%$ when drug response was $15 \pm 5 \mathrm{mmHg}$. Variation in measurement ( $\left.\sigma_{\text {meas }}\right)$ had no effect on the maximal proportion achieving the $\mathrm{MSBP}$ target, since this error is not apparent to the observer. With multiple titration steps and at higher initial tSBP, we found an inverse relationship between measurement error and the proportion achieving $\mathrm{mSBP}<140 \mathrm{mmHg}$ (Table 1).

The relative effects of the input parameters on the proportion of individuals who achieved target (tSBP $<140 \mathrm{mmHg}$ ) are presented in Figure 3. Initial tSBP, measurement error and drug response all influenced the proportion of the population who would achieve target should the simulation be run infinitum. Measurement error accounted for a difference of almost 30\% ( $\sigma_{\text {meas }} 5$ versus 15 $\mathrm{mmHg}$ ) at initial tSBP $170 \mathrm{mmHg}$, compared to $<10 \%$ at $150 \mathrm{mmHg}$. When measurement error was reduced to below that achieved in clinical practice $\left(\sigma_{\text {meas }} 5\right.$ $\mathrm{mmHg}$ ) the proportion who failed to achieve tSBP $<140 \mathrm{mmHg}$ remained high at approximately $30 \%$ for initial tSBP $170 \mathrm{mmHg}$. The proportion who failed to achieve control on three drugs is shown in Table 1.

Drug response influenced both the number of steps required to achieve tSBP $<140$ $\mathrm{mmHg}$ and the maximal proportion of the population who achieved the target. Where drug response was low (drug eff $5 \mathrm{mmHg}$ ), for an initial tSBP $170 \mathrm{mmHg}$ approximately $40 \%$ achieved target after nine steps (a hypothetical treatment outcome), compared to approximately $75 \%$ where drug response was high (drug eff $15 \mathrm{mmHg}$ ) (Figure 3). Variation in drug response ( $\sigma_{\text {drug }}$ ) also influenced the proportion reaching tSBP target but accounted for $<10 \%$ difference for all populations. 
Due to the impact of measurement error on achieving target tSBP we next considered how repeated sets of readings might mitigate this. In the first instance we simulated a second, independent measurement at each step when mSBP 120$150 \mathrm{mmHg}$. The second measurement in this 'at risk population' was then used to determine the outcome (i.e. addition of another drug or exit simulation) for that individual. Figure 4 shows that when the first and second measurements had the same error ( $\sigma_{\text {meas }} 15 \mathrm{mmHg}$ ) there was an increase in the proportion of individuals with controlled tSBP (49.9\% versus $60.8 \%$ for initial tSBP $170 \mathrm{mmHg}$ ) and a decrease in individuals with $\mathrm{tSBP} \geq 140 \mathrm{mmHg}$ across the range of initial tSBP. When a method of SBP measurement with a lower measurement error was used for the second measurement $\left(\sigma_{\text {meas }} 5 \mathrm{mmHg}\right.$ ), the proportion of individuals with controlled tSBP increased further (78.0\%) with a corresponding decrease in over- and undertreatment.

\section{Discussion}

The results of our simulations indicate that a large proportion of hypertensive individuals in whom SBP is thought to be at target following an initial drug titration, are in fact over- or undertreated. These individuals remain at either an increased risk of cardiovascular events or have an increased risk of side-effects from excessive medication. For example, in individuals with stage two hypertension $<65 \%$ are controlled to within the target window (tSBP 120-139 mmHg) when clinically relevant base case parameters are input to the model.

The model enabled us to interrogate which parameters have the greatest influence on both the number of drugs required to achieve tSBP control, and the proportion of individuals in whom control is actually achieved. Several important findings have emerged. Firstly, the mean drug response (drug $\mathrm{eff}_{\text {f }}$ and/or standard deviation of this response $\left(\sigma_{\text {drug }}\right.$ ) principally influences the number of titration steps required to achieve $\mathrm{mSBP}<140 \mathrm{mmHg}$, rather than define the upper limit. Where the number of titration steps are unlimited (a hypothetical scenario), all individuals will eventually achieve $\mathrm{mSBP}<140 \mathrm{mmHg}$. However, each titration step provides an opportunity for incorrect classification of SBP ( $\mathrm{mSBP}<140 \mathrm{mmHg}$, despite tSBP $\geq 140 \mathrm{mmHg}$ ) and it is 
for this reason that the proportion of individuals achieving target was lower when drug $_{\text {eff }}$ was reduced.

Secondly, the variation in SBP measurement ( $\left.\sigma_{\text {meas }}\right)$ had no effect on the maximal proportion achieving $\mathrm{mSBP}<140 \mathrm{mmHg}$ as the clinician is blinded to measurement error. However, a large effect was observed once tSBP values were analysed, with a lower proportion achieving tSBP $<140 \mathrm{mmHg}$ at higher initial tSBPs. Again, this represents the number of titration steps and thus the number of opportunities for measurement error. In our simulation, it is measurement error that determines most of the misclassification where individuals are thought to be controlled based on mSBP, but tSBP lies outside of the target window.

Thirdly, the proportion of individuals misclassified can be reduced by an independent, second measurement when mSBP lies within a range that is 'at risk' from measurement error. The proportion misclassified was reduced further when the second measurement had lower variation than the first.

The composite of our analyses demonstrate that an individual is most likely to have their SBP misclassified if they have a high initial tSBP, receive drugs of low efficacy (or suboptimal adherence), and have titration decisions based on a single SBP measurement with high variability. The model allows consideration of remedial measures to reduce the number of subjects misclassified. Options to reduce measurement error include the use of single measurement events with increased precision, or repeated measurements that are averaged over longer periods (e.g. oscillometric home BP monitoring or the use of wearable technology). Such an approach could be applied selectively to individuals with measurements close to threshold values to reduce the measurement burden, or to those who exhibit high variability to improve accuracy. Interestingly, the simulation suggests that a small improvement in measurement error (10 versus $15 \mathrm{mmHg}$ ) will have negligible impact on misclassification, and that an error level not currently achieved with any clinical technique $(5 \mathrm{mmHg})$ is required. 
However even with an accuracy greater than that usually achieved in clinical practice, a substantial proportion of individuals will still be misclassified and receive suboptimal management. An alternative approach would be to recognise that as a high proportion of individuals are undertreated due to misclassification, the target window could be lowered to reduce this number. This would result in fewer individuals with uncontrolled BP but a larger number with relatively low BP. Whether a low BP might be associated with increased morbidity and/or mortality is uncertain and such a strategy would have to be formally tested in clinical trials. The challenge of balancing SBP targets with measurement method can be observed in the varying response by guideline committees to the results from the Systolic Blood Pressure Intervention Trial (SPRINT) $[1,6,7,26]$. The SBP target selected for this simulation is consistent with UK primary prevention guidelines, but the findings are applicable to alternative targets and healthcare systems [27].

It is important to stress that the simulation exercise presented here is primarily for illustrative purposes since there are several assumptions and simplifications inherent to the model. The simulation is designed to investigate the number of treatment steps required to achieve $\mathrm{mSBP}<140 \mathrm{mmHg}$ and so does not consider options for antihypertensive de-escalation as a response to either medication side-effects or overtreatment. The limited time horizon does not consider long-term SBP control. However, the proportion of individuals requiring three of more drugs to achieve $\mathrm{mSBP}<140 \mathrm{mmHg}$ with clinically relevant parameters, corresponds to the prevalence of resistant hypertension both globally and in the UK $[28,29]$. The main findings of this study would remain true if a more sophisticated simulation was used whereby drug response varied depending on SBP and the number of titrations [11].

The strength of the present simulation is that it allows the exploration of various management scenarios as a function of the burden of achieving more accurate measurements. Such an approach could inform the best strategies to be tested in clinical trials and provide more accurate data for health economic analyses. A full health economics analysis would require quantification of the relative burden of improving measurement precision and the risk related to poorly controlled 
hypertension. Modern technology, such as a wearable wrist monitor, if sufficiently free from systematic bias, could potentially improve estimations of tSBP through the capacity to take high numbers of BP readings which would be sampled from the full range of potential measurement error. Such an approach would place little or no burden on patients or healthcare staff and could have a major impact on hypertension control.

Our approach to simulating uncertainty can also be applied to questions which have either not been fully addressed in clinical trials or for where there remains uncertainty in interpretation of the evidence. One prominent example is the use of initial dual antihypertensive therapy which is recommended by both European and US guidelines $[6,7]$. In contrast, the recent UK guidelines were unable to recommend this approach due to a lack of cardiovascular outcome data [27]. By combining simulated tSBP data with a cardiovascular outcome model (in which benefit is proportion to SBP reduction [11]) it may be possible to add clarity to the issue. Furthermore, such an approach could be extended to situations which have not been addressed in cardiovascular outcome studies such as initial triple antihypertensive therapy, decision making based on repeated measures, combinations of halfdose/full-dose antihypertensives or novel polypill formulations.

In conclusion, Monte Carlo simulations have identified measurement error as the major determinant for individuals trying to achieve blood pressure control during initial medication titration. Strategies to reduce measurement error should be tested in pragmatic clinical trials. 


\section{Sources of Funding}

This work was performed as part of the AIM HY (Ancestry and biological Informative Markers in stratification of Hypertension, MR/M016560/1) stratified medicines programme in hypertension funded by the Medical Research Council and The British Heart Foundation. We also acknowledge support from the Department of Health via a National Institute for Health Research (NIHR) Biomedical Research Centre and Clinical Research Facility award to Guy's \& St Thomas' NHS Foundation Trust in partnership with King's College London, and the NIHR Biomedical Research Centre at South London and Maudsley NHS Foundation Trust and King's College London. Lorenzo Zanisi was funded through a DISCnet placement scheme organized through the University of Portsmouth and King's College London. 


\section{References:}

1. Yannoutsos, A., R. Kheder-Elfekih, J.M. Halimi, M.E. Safar, and J. Blacher, Should blood pressure goal be individualized in hypertensive patients? Pharmacol Res, 2017. 118: p. 53-63.

2. Lim, S.S., T. Vos, A.D. Flaxman, G. Danaei, K. Shibuya, H. Adair-Rohani, et al., A comparative risk assessment of burden of disease and injury attributable to 67 risk factors and risk factor clusters in 21 regions, 19902010: a systematic analysis for the Global Burden of Disease Study 2010. Lancet, 2012. 380(9859): p. 2224-60.

3. Forouzanfar, M.H., P. Liu, G.A. Roth, M. Ng, S. Biryukov, L. Marczak, et al., Global Burden of Hypertension and Systolic Blood Pressure of at Least 110 to $115 \mathrm{~mm} \mathrm{Hg}$, 1990-2015. JAMA, 2017. 317(2): p. 165-182.

4. Lewington, S., R. Clarke, N. Qizilbash, R. Peto, R. Collins, and C. Prospective Studies, Age-specific relevance of usual blood pressure to vascular mortality: a meta-analysis of individual data for one million adults in 61 prospective studies. Lancet, 2002. 360(9349): p. 1903-13.

5. Ettehad, D., C.A. Emdin, A. Kiran, S.G. Anderson, T. Callender, J. Emberson, et al., Blood pressure lowering for prevention of cardiovascular disease and death: a systematic review and meta-analysis. Lancet, 2016. 387(10022): p. 957-967.

6. Whelton, P.K., R.M. Carey, W.S. Aronow, D.E. Casey, Jr., K.J. Collins, C. Dennison Himmelfarb, et al., 2017 ACC/AHA/AAPA/ABC/ACPM/AGS/APhA/ASH/ASPC/NMA/PCNA Guideline for the Prevention, Detection, Evaluation, and Management of High Blood Pressure in Adults: Executive Summary: A Report of the American College of Cardiology/American Heart Association Task Force on Clinical Practice Guidelines. Circulation, 2018. 138(17): p. e426-e483.

7. Williams, B., G. Mancia, W. Spiering, E. Agabiti Rosei, M. Azizi, M. Burnier, et al., 2018 ESC/ESH Guidelines for the management of arterial hypertension: The Task Force for the management of arterial hypertension of the European Society of Cardiology and the European Society of Hypertension: The Task Force for the management of arterial hypertension of the European Society of Cardiology and the European Society of Hypertension. J Hypertens, 2018. 36(10): p. 1953-2041.

8. Deary, A.J., A.L. Schumann, H. Murfet, S.F. Haydock, R.S. Foo, and M.J. Brown, Double-blind, placebo-controlled crossover comparison of five classes of antihypertensive drugs. J Hypertens, 2002. 20(4): p. 771-7.

9. Law, M.R., N.J. Wald, J.K. Morris, and R.E. Jordan, Value of low dose combination treatment with blood pressure lowering drugs: analysis of 354 randomised trials. BMJ, 2003. 326(7404): p. 1427.

10. Mancia, G., M. Bombelli, R. Facchetti, F. Madotto, G. Corrao, F.Q. Trevano, et al., Long-term prognostic value of blood pressure variability in the general population: results of the Pressioni Arteriose Monitorate e Loro Associazioni Study. Hypertension, 2007. 49(6): p. 1265-70.

11. Law, M.R., J.K. Morris, and N.J. Wald, Use of blood pressure lowering drugs in the prevention of cardiovascular disease: meta-analysis of 147 randomised trials in the context of expectations from prospective epidemiological studies. BMJ, 2009. 338: p. b1665. 
12. Eguchi, K., S. Hoshide, J.E. Schwartz, K. Shimada, and K. Kario, Visit-to-visit and ambulatory blood pressure variability as predictors of incident cardiovascular events in patients with hypertension. Am J Hypertens, 2012. 25(9): p. 962-8.

13. Stevens, S.L., S. Wood, C. Koshiaris, K. Law, P. Glasziou, R.J. Stevens, et al., Blood pressure variability and cardiovascular disease: systematic review and meta-analysis. BMJ, 2016. 354: p. i4098.

14. Kallioinen, N., A. Hill, M.S. Horswill, H.E. Ward, and M.O. Watson, Sources of inaccuracy in the measurement of adult patients' resting blood pressure in clinical settings: a systematic review. J Hypertens, 2017. 35(3): p. 421441.

15. Hwang, K.O., A. Aigbe, H.H. Ju, V.C. Jackson, and E.W. Sedlock, Barriers to Accurate Blood Pressure Measurement in the Medical Office. J Prim Care Community Health, 2018. 9: p. 2150132718816929.

16. Morcos, R.N., K.J. Carter, F. Castro, S. Koirala, D. Sharma, and H. Syed, Sources of Error in Office Blood Pressure Measurement. J Am Board Fam Med, 2019. 32(5): p. 732-738.

17. Turner, M.J., L. Irwig, A.J. Bune, P.C. Kam, and A.B. Baker, Lack of sphygmomanometer calibration causes over- and under-detection of hypertension: a computer simulation study. J Hypertens, 2006. 24(10): p. 1931-8.

18. Turner, M.J. and J.M. van Schalkwyk, Blood pressure variability causes spurious identification of hypertension in clinical studies: a computer simulation study. Am J Hypertens, 2008. 21(1): p. 85-91.

19. Rodrigues Filho, B.A., R.F. Farias, and W. Dos Anjos, Evaluating the impact of measurement uncertainty in blood pressure measurement on hypertension diagnosis. Blood Press Monit, 2018. 23(3): p. 141-147.

20. Bonate, P.L., A brief introduction to Monte Carlo simulation. Clin Pharmacokinet, 2001. 40(1): p. 15-22.

21. Shankar, F., S. Mei, M. Huertas-Company, J. Moreno, F. Fontanot, P. Monaco, et al., Environmental dependence of bulge-dominated galaxy sizes in hierarchical models of galaxy formation. Comparison with the local Universe. MNRAS, 2014. 439: p. 3189-3212.

22. Shankar, F., S. Buchan, A. Rettura, V.R. Bouillot, J. Moreno, R. Licitra, et al., Avoiding Progenitor Bias: The Structural and Mass Evolution of Brightest Group and Cluster Galaxies in Hierarchical Models since $z<1$. The Astrophysical Journal, 2015. 802(2): p. 73.

23. Zanisi, L., F. Shankar, A. Lapi, N. Menci, M. Bernardi, C. Duckworth, et al., Galaxy sizes and the galaxy-halo connection - I. The remarkable tightness of the size distributions. Monthly Notices of the Royal Astronomical Society, 2020. 492(2): p. 1671-1690.

24. Williams, B., L.H. Lindholm, and P. Sever, Systolic pressure is all that matters. Lancet, 2008. 371(9631): p. 2219-21.

25. Accord Study Group, W.C. Cushman, G.W. Evans, R.P. Byington, D.C. Goff, Jr., R.H. Grimm, Jr., et al., Effects of intensive blood-pressure control in type 2 diabetes mellitus. N Engl J Med, 2010. 362(17): p. 1575-85.

26. Sprint Research Group, J.T. Wright, Jr., J.D. Williamson, P.K. Whelton, J.K. Snyder, K.M. Sink, et al., A Randomized Trial of Intensive versus Standard Blood-Pressure Control. N Engl J Med, 2015. 373(22): p. 2103-16. 
27. Boffa, R.J., M. Constanti, C.N. Floyd, A.S. Wierzbicki, and C. Guideline, Hypertension in adults: summary of updated NICE guidance. BMJ, 2019. 367: p. 15310.

28. Achelrod, D., U. Wenzel, and S. Frey, Systematic review and meta-analysis of the prevalence of resistant hypertension in treated hypertensive populations. Am J Hypertens, 2015. 28(3): p. 355-61.

29. Sinnott, S.J., L. Smeeth, E. Williamson, and I.J. Douglas, Trends for prevalence and incidence of resistant hypertension: population based cohort study in the UK 1995-2015. BMJ, 2017. 358: p. j3984. 
Tables

Table 1: The impact of measurement error ( $\sigma_{\text {meas }}$ ) on achieving target blood pressure for individuals taking three antihypertensive medications.

\begin{tabular}{|l|c|c|c|c|c|c|}
\hline \multirow{2}{*}{} & \multicolumn{2}{|c|}{ Initial tSBP } & \multicolumn{2}{c|}{ Initial tSBP } & \multicolumn{2}{c|}{ Initial tSBP } \\
& \multicolumn{2}{|c|}{$150 \mathrm{mmHg}$} & \multicolumn{2}{c|}{$160 \mathrm{mmHg}$} & \multicolumn{2}{c|}{$170 \mathrm{mmHg}$} \\
\hline$\sigma_{\text {meas }}$ & $\mathrm{mSBP}$ & tSBP & $\mathrm{mSBP}$ & tSBP & $\mathrm{mSBP}$ & tSBP \\
$(\mathbf{m m H g})$ & $\geq 140$ & $\geq 140$ & $\geq 140$ & $\geq 140$ & $\geq 140$ & $\geq 140$ \\
& $\mathbf{m m H g}$ & $\mathbf{m m H g}$ & $\mathrm{mmHg}$ & $\mathrm{mmHg}$ & $\mathrm{mmHg}$ & $\mathrm{mmHg}$ \\
\hline $\mathbf{5}$ & $1.2 \%$ & $15.1 \%$ & $13.2 \%$ & $27.1 \%$ & $48.3 \%$ & $56.0 \%$ \\
\hline $\mathbf{1 0}$ & $1.8 \%$ & $20.4 \%$ & $13.3 \%$ & $39.6 \%$ & $42.2 \%$ & $69.2 \%$ \\
\hline $\mathbf{1 5}$ & $2.8 \%$ & $22.5 \%$ & $13.0 \%$ & $46.7 \%$ & $35.4 \%$ & $68.5 \%$ \\
\hline
\end{tabular}

Simulation parameters: drug eff $_{10} \mathrm{mmHg} ; \sigma_{\text {drug }} 5 \mathrm{mmHg}$; $\sigma_{\text {meas }} 5-15 \mathrm{mmHg}$.

[mSBP: measured systolic blood pressure; tSBP: true systolic blood pressure] 


\section{Captions for Figures}
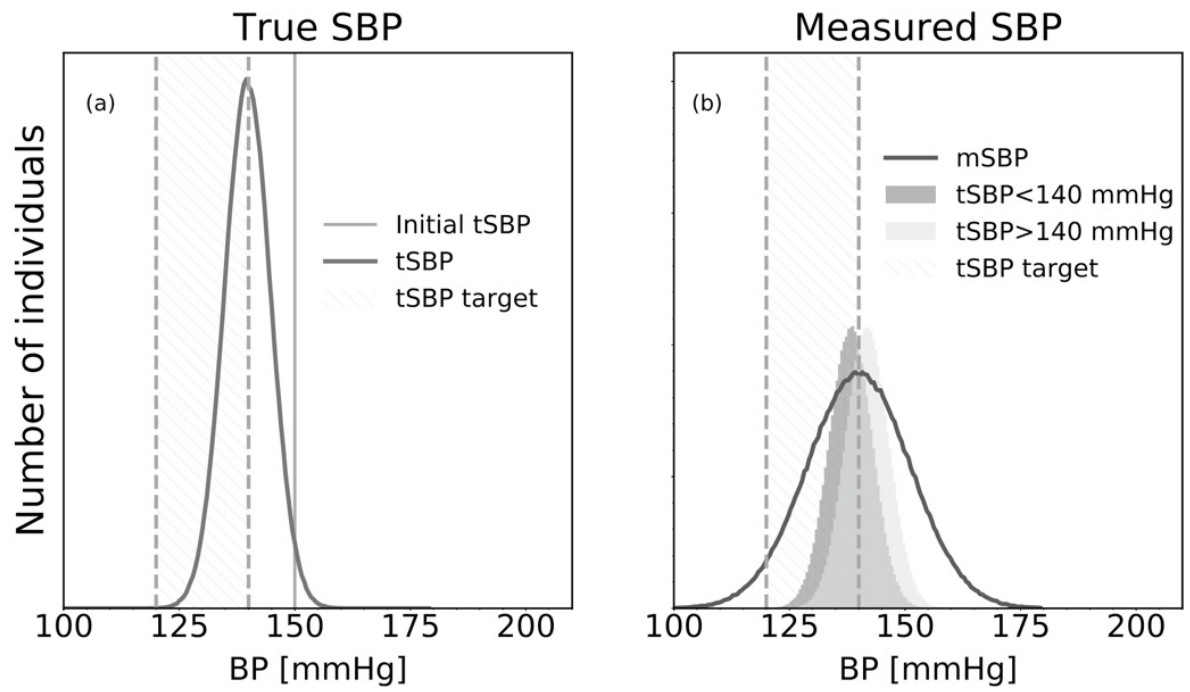

Figure 1: Distribution of (A) true systolic blood pressure (tSBP) and (B) measured systolic blood pressure (mSBP) after a single treatment step. Simulation conducted with an initial tSBP $150 \mathrm{mmHg}$, drug response (drugeff $\left.\pm \sigma_{\text {drug }}\right) 10 \pm 5 \mathrm{mmHg}$ and standard deviation of measurement ( $\left.\sigma_{\text {meas }}\right) 10 \mathrm{mmHg}$. 

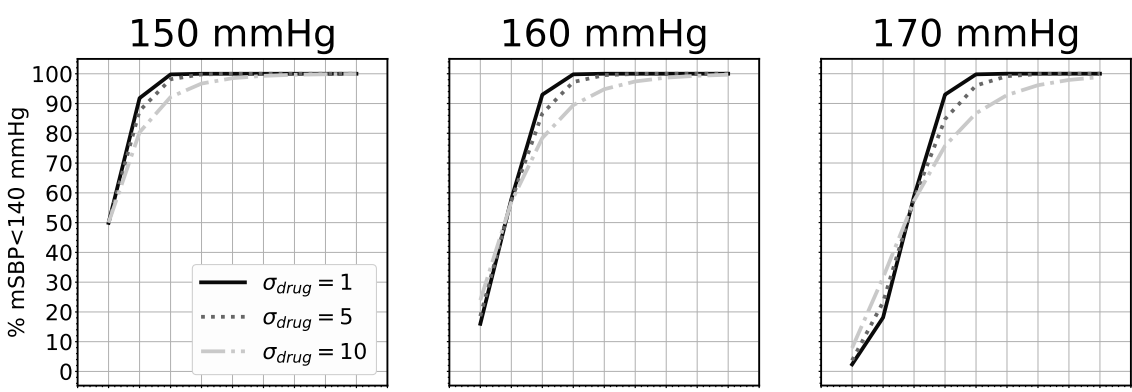

Fixed parameters:

$\sigma_{\text {meas }}=10 \mathrm{mmHg}$ drug $_{\text {eff }}=10 \mathrm{mmHg}$
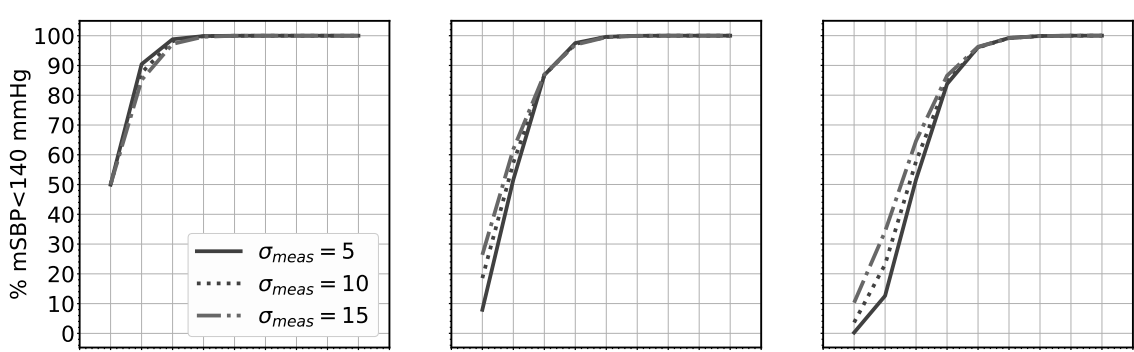

Fixed parameters:

$\sigma_{\text {drug }}=5 \mathrm{mmHg}$ drug $_{\text {eff }}=10 \mathrm{mmHg}$
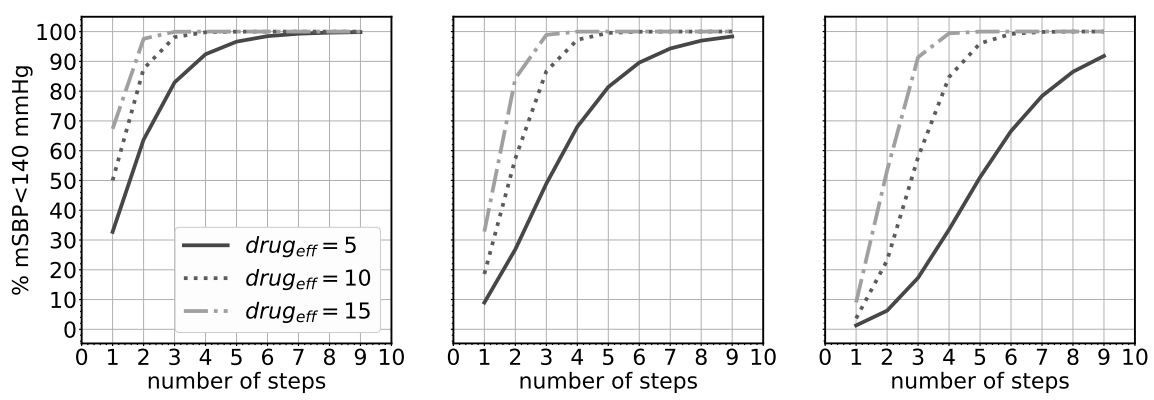

Fixed parameters:

$\sigma_{\text {drug }}=5 \mathrm{mmHg}$

$\sigma_{\text {meas }}=10 \mathrm{mmHg}$

Figure 2: Proportion of individuals achieving a measured systolic blood pressure $(\mathrm{mSBP})<140 \mathrm{mmHg}$ with increasing drug titration.

Simulation inputs were varied based on initial true SBP (top of figure), drug response

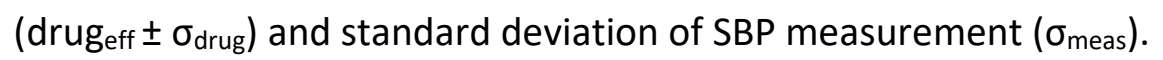



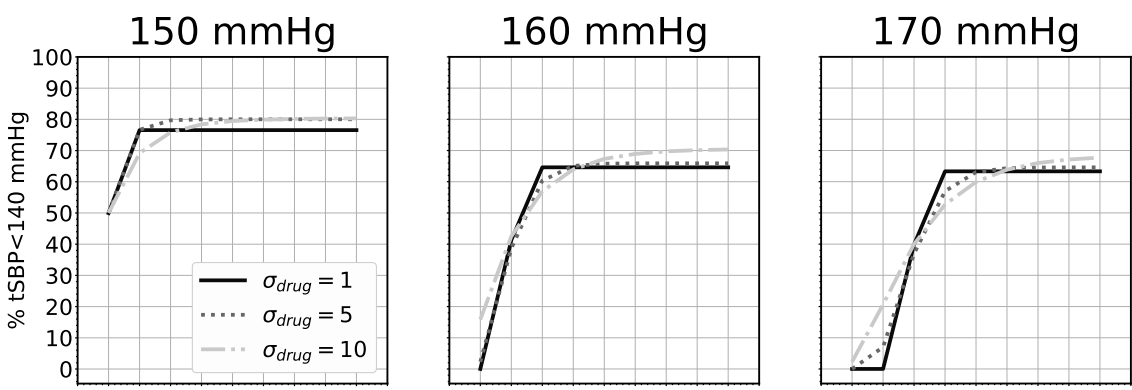

Fixed parameters:

$\sigma_{\text {meas }}=10 \mathrm{mmHg}$ drug $_{\text {eff }}=10 \mathrm{mmHg}$
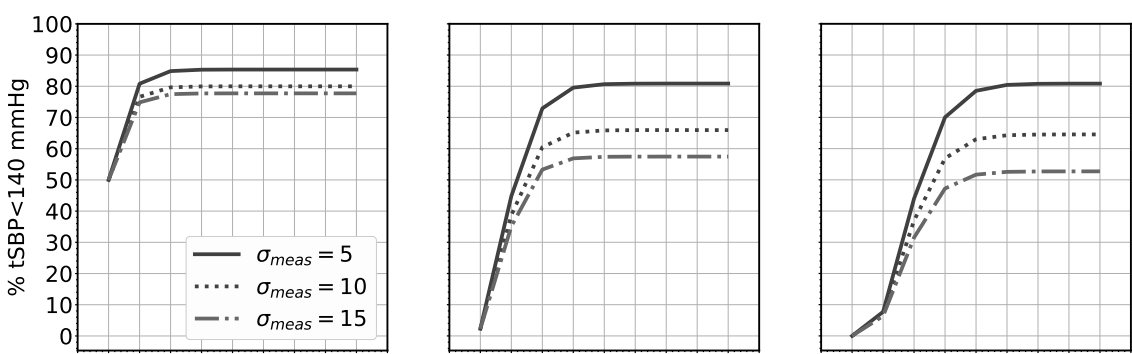

Fixed parameters:

$\sigma_{\text {drug }}=5 \mathrm{mmHg}$ drug $_{\text {eff }}=10 \mathrm{mmHg}$
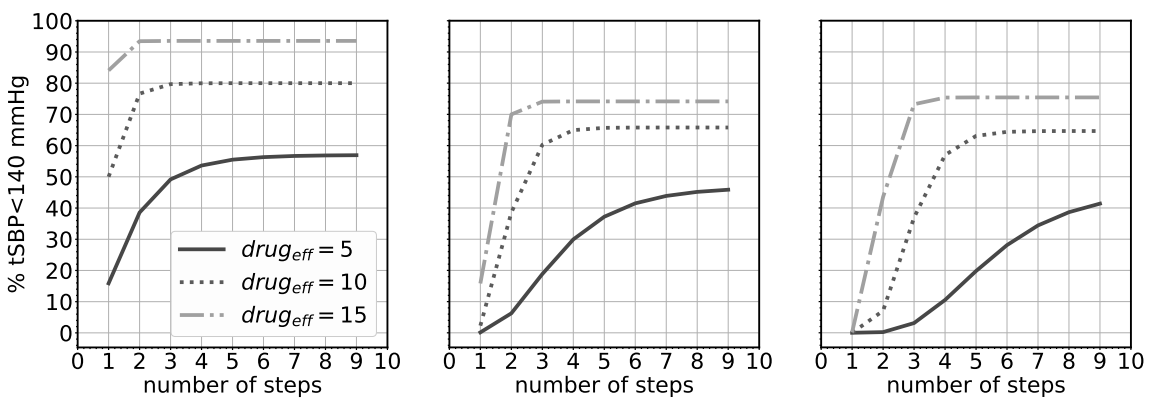

Fixed parameters:

$\sigma_{\text {drug }}=5 \mathrm{mmHg}$

$\sigma_{\text {meas }}=10 \mathrm{mmHg}$

Figure 3: Proportion of individuals achieving a true systolic blood pressure (tSBP) $<140 \mathrm{mmHg}$ with increasing drug titration.

tSBP values calculated once $\mathrm{mSBP}<140 \mathrm{mmHg}$ and subject had exited the simulation. Simulation inputs were varied based on initial t SBP (at top of figure), drug response (drug eff $\pm \sigma_{\text {drug }}$ ) and standard deviation of SBP measurement ( $\sigma_{\text {meas }}$ ). 
(a)

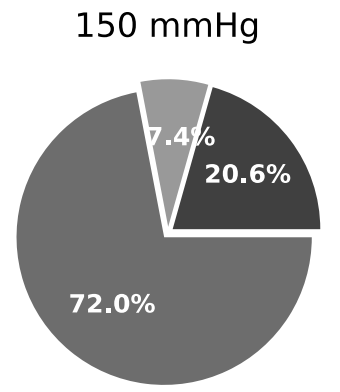

(b)

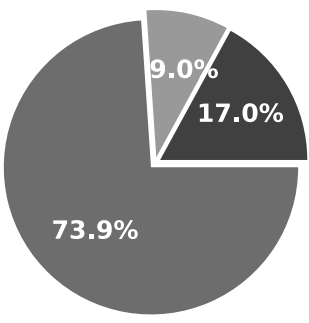

(c)

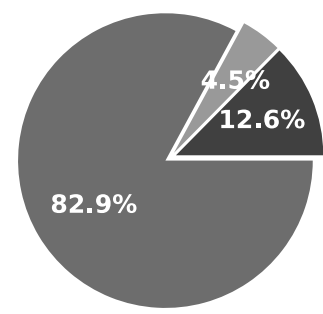

$160 \mathrm{mmHg}$
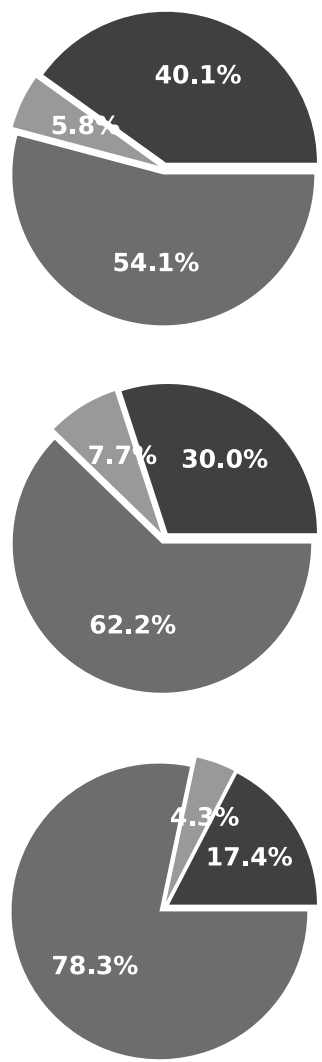

$170 \mathrm{mmHg}$
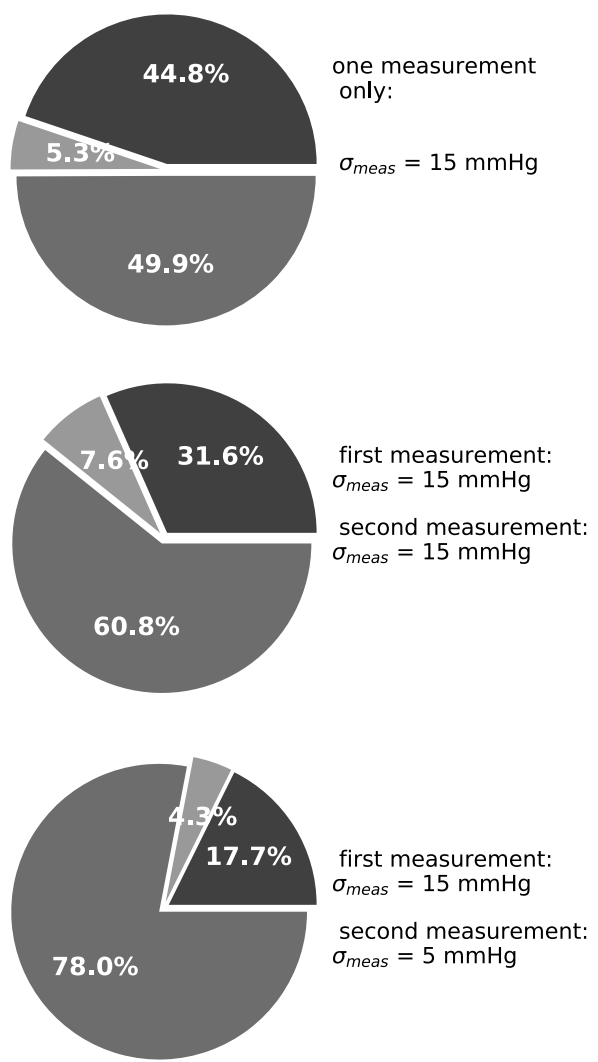

Undertreated (tSBP>140 $\mathrm{mmHg}$ )

Overtreated (tSBP $<120 \mathrm{mmHg}$ )

Controlled (tSBP $120-139 \mathrm{mmHg}$ )

Figure 4: Classification of true systolic blood pressure (tSBP) for individuals exiting the simulation based on $(A)$ single $m S B P,(B)$ repeated measurement when first mSBP 120-150 mmHg, and (C) when second measurement is repeated with greater precision ( $\sigma_{\text {meas }} 5 \mathrm{mmHg}$ ) when first mSBP $120-150 \mathrm{mmHg}$. Analyses performed for different initial tSBP (top of figure). The response to each titration step was fixed at $10 \pm 5 \mathrm{mmHg}$ (drug eff $\pm \sigma_{\text {drug }}$ ). 